\title{
Identification of Antimastits Componenets in Boerhavia diffusa as an Inhibitor of Staphalococus aureus Monofunctional Glycosyltransferase, Causing Bovine mastitis (An Insilico Approach)
}

\author{
B. SRUTHY ${ }^{1}$, M. S. LATHA ${ }^{2 *}$ and SHERLY. P. ANAND ${ }^{3}$ \\ 'Department of Chemistry, Sree Narayana College of Arts and Science, Kollam, Kerala, India. \\ ${ }^{2}$ Department of Chemistry, Sree Narayana College, Neduvaramcode P.O. Chengannur, \\ Alappuzha, Kerala, India. \\ ${ }^{3}$ Department of Zoology, TK Madhava Memmorial College of Arts and Science, \\ Nangiarkulangara, Alappuzha, Kerala, India. \\ *Corresponding author E-mail: lathams2014@gmail.com \\ http://dx.doi.org/10.13005/ojc/340264
}

(Received: December 08, 2017; Accepted: January 20, 2018)

\begin{abstract}
Bovine mastitis is an infection of cattle leading to a huge reduction in milk production that causes severe economic loss in dairy industry across the world. Causative of the disease includes bacteria, virus and non-bacterial pathogens. Among these, Staphalococus aureus is the common cause of mastitis and is highly resistant to the most routinely used antibiotics. So current antibiotic therapy has shown limited efficacy. The crude extract of locally available medicinal plant Boerhavia diffusa is used traditionally against mastitis and is found to be highly effective. The objective of the study is the identification of the phytochemicals in Boeravia diffusa responsible for the antimastitis activity by insilico docking analysis using Schrodinger Suit v 9.2. 20 phytochemicals in Boerhavia diffusa were selected for docking studies based on ADMET properties. The high resolution crystal structure of the target receptor protein of Staphalococus aureus was retrieved from PDB. Structure of the phytochemicals and the most commonly used antibiotic against Bovine mastitis were selected from PUB CHEM NCBI. The phytochemicals, Boeravinone A, B, C, D, $\mathrm{E}$ and $\mathrm{F}$ from Boehravia diffusa showed good docking scores and better interaction with the active sites of the target proteins used for the evaluation than the most commonly used commercially available drug. Results of this study are important for the designing of novel drugs for the treatment of mastitis.
\end{abstract}

Keywords: Staphalococus aureus monofunctional glycosyltransferase, Bovine mastitis, Docking, Boerhavia diffusa, Qik prop. 


\section{INTRODUCTION}

Bovine mastitis is a kind of inflammation of the udder of cattle due to wounds or scars. It is associated with the presence of infectious agents as bacteria, Bovine Herpes virus, non bacterial pathogens like mycoplasmas, fungi, yeast and chalmydia ${ }^{1-2}$. Among these, bacterial infections are most common. Bovine mastitis reduces the quantity of casein, lactoferrin and potassium in milk. As the major protein, casein in milk deteriorates, the calcium level in milk also decreases. During processing and storage also the milk protein undergo deterioration ${ }^{3}$. Milk from affected animals shows very high somatic cell count which lowers the quality of milk ${ }^{4}$. Hence, Bovine mastitis causes severe economic losses in dairy industry. ${ }^{5}$

Current methods used in the control of Bovine mastitis are based on antibiotic therapy. The commonly used antibiotics are pencillins and semisynthetic pencillin derivatives targeting the pencillin binding proteins of Staphalococus aureus. Pencillin binding proteins or DD- transpeptidase form bonds between the oligopeptide crosslinks in peptidoglycan ${ }^{6}$. Inhibition of peptidoglycan synthesis leads to bacterial cell lysis ${ }^{7}$. Commonly used antibiotics target the DD-transpeptidase and inhibit peptidoglycan synthesis there by leading to bacterial cell lysis. But mutations in genes coding for transpeptidases leads to reduced interaction with antibiotic results in the generation of antibiotic resistance in Staphalococus aureus ${ }^{8}$. This is the main reason for the decreased antibiotic efficacy in the treatment of Bovine mastitis. Monofunctional glycosyl transferase ${ }^{1}$ is an alternate potent target of Staphalococus aureus, the inhibition of which also leads to bacterial cell lysis.

The immunomodulator compounds derived from medicinal plants has potent application in controlling mastitis. Boerhavia diffusa is one of the medicinal plants in the whole or its peculiar parts have enormous medicinal properties ${ }^{9}$. Farmers of the Southern region of Kerala uses the crude extract of the plant as a whole as a medicine for mastitis from their traditional knowledge.

\section{Methodology \\ Bioinformatics analysis \\ Preparation of protein}

Crystal structure of the target proteins of Staphalococus aureus Monofunctional Glycosyltransferase was obtained from RCSB Protein Data Bank (PDB ID: 3HZS) ${ }^{10}$. Automatically imported PDB files from the RCSB PDB website to the Maestro working interface. ${ }^{10-12}$ Optimized the protein's hydrogen bond network by means of a systematic, cluster-based approach, which greatly decreased preparation times and then performed a restrained minimization that allows hydrogen atoms to be freely minimized. Here the crystal structure of the target protein was complexed with a ligand. So sitemap creation was exempted.

\section{Ligand preparation}

Twenty phytochemicals present in Boerahavia diffusa ${ }^{13}$ were selected to find out the inhibitory activity towards the target protein. (Betasitosterol, BoeravinoneA, B, C, D, E, F, Hexacosanoic acid, Tetracosanoic acid, Sitosterylferulate, Liriodendrin, Arachidic acid, Triacondanol, Palmitic acid, Stearic acid, Sitosterylpalmitate, Hentriacontane, Sitosterylester, Ursolic acid). The structure of the phytochemicals and commonly used commercially available antibiotic Cloxacillin were downloaded from pub chem in the (.sdf) format. These ligands were subjected to ligand preparation using the ligand preparation wizard (ligprep) of Schrodinger software in the Maestro interface12 ${ }^{11-12}$. One low energy conformation was generated. The ligprep ligands were used for the Docking analysis.

\section{Docking studies}

The compounds were screened by Schrodinger docking software to study inhibitors of the target protein. Grid generation was done using the centroid of workspace ligand R0 48-8071. The rigid receptor docking using the Glide program was carried out against the target protein with the set of ligands. The mode of docking was selected as XP (Extra precision) for a high docking accuracy. The glide docking was carried out for the minimised protein $^{11,12,14}$. 


\section{Ligand interaction study}

The ligand molecules fit in to the active binding sites of the protein molecules by means of some interactions. The interactions include hydrogen bonding, pi-pi stalking interactions and pi-pi cation interactions. A detailed information of various interactions of the ligands with aminoacid residues pointing the type of bonds, bond length and various angles were studied using this option.

\section{ADMET properties prediction}

The bioactive compounds from Boerhavia diffusa were checked for their ADMET properties using Qik prop module. ${ }^{14-15}$ QikProp helped in analyzing the pharmacokinetics and pharmacodynamics of the ligand by accessing the drug likeness.

\section{RESULTS AND DISCUSSION}

Boeravia diffusa is a renowned medicinal plant due to its therapeutic potential. Aqueos leaf extract of Boeravia diffusa had hypoglycaemic effect ${ }^{16}$ and nutritive properties ${ }^{17}$. Ethanolic extract of Boeravia diffusa leaves had antistress, adoptogenic, immunopotentiating ${ }^{18}$ and anti-inflammatory activity ${ }^{19}$. Antibacterial activity of methanolic and ethanolic extracts of Boeravia diffusa leaves against both gram positive and Gram-negative bacteria were reported. ${ }^{20} \mathrm{Also}$ aqueous and ethanolic extracts of Boeravia diffusa had antibacterial activity on E.Coli, Staphalococus aureus and $P$. aeruginosa. ${ }^{21}$

Present study revealed the phytochemicals in Boerhavia diffusa which are responsible for the antimastitis activity by inhibiting the most potent drug target in Staphalococus aureus. Table 1 shows the docking scores, number of hydrogen bonds, interacting amino acid residues in the active site of protein and hydrogen bond length. Table 2 explains the drug likeness of phytochemicals predicted by QikProp simulation. Fig. 1 and Fig. 2 shows the $2 D$ and $3 D$ interactions of the ligands with the target protein.

From the results obtained, it has been understood that there exist excellent binding interactions between phytochemicals in Boerhavia diffusa and target proteins (PDB ID:4HZS) compared to the commercial drug (cloxacillin) with good binding scores. In the protein monofunctional glycosyltransferase with PDB ID: $3 \mathrm{HZS}$, the $110^{\text {th }} \mathrm{PHE}, 136^{\text {th }} \mathrm{GLN}$ and $130^{\text {th }} \mathrm{GLN}$ aminoacid residues forms the binding pocket. The phytochemical Boeravinone $\mathrm{E}$ shows a binding score $-6.151 \mathrm{kCal} / \mathrm{mol}$ and forms three hydrogen bonds with the aminoacid residues in the binding pocket. The hydroxyl group in the $11^{\text {th }}$ position of the chromeno chromon nucleus forms a hydrogen bond with the amino acid residue $110^{\text {th }} \mathrm{PHE}$ (bond length-2.04799, Acceptor Angle-143.297 and Donor angle-138.404) Similarly, the hydroxyl group in the $9^{\text {th }}$ position of the chromeno chromon nucleus of Boeravinone $E$ forms a hydrogen bond with $130^{\text {th }} \mathrm{GLY}$ residue.(Bond length-2.18231, Acceptor angle-143.17 and Donor angle-119.565) There is a side to side hydrogen bond between the ketonic oxygen of Boeravinone $E$ with $136^{\text {th }}$ GLN amino acid residue.(bond length-2.15438, Donor angle-162.547.

The phytochemical Boeravinone $\mathrm{D}$ exhibit three hydrogen bonding interactions with a docking score $-6.013 \mathrm{kCal} / \mathrm{mol}$. The two hydroxyl groups in the $11^{\text {th }}$ and $9^{\text {th }}$ positions of the chromeno chromon nucleus forms two hydrogen bonds with the $130^{\text {th }}$ GLY and $110^{\text {th }}$ PHE aminoacid residues.(Bond length-2.11767 and 1.89519, Acceptor angle-170.817 and 151.217, Donor angle-166.252 and 138.416) respectively. The ketonic oxygen of the chromon nucleus forms a side to side hydrogen bond with $136^{\text {th }} \mathrm{GLN}$. (Bond length-2.09866, Donor angle-165.062).

Boeravinone $\mathrm{F}$ forms four hydrogen bonds with a docking score -5.629 . The hydroxyl group in the $11^{\text {th }}$ position forms a hydrogen bond with the aminoacid residue $136^{\text {th }} \mathrm{GLN}$ (Bond length2.14702, acceptor angle-170.600 and Donor angle-149.579). This hydroxyl group also forms another hydrogen bond with $110^{\text {th }} \mathrm{PHE}$ (Bond length-1.88670,acceptor angle-161.487 and Donor angle-156.617. Ketonic oxygen forms a side to side hydrogen bond with $136^{\text {th }}$ GLN aminoacid residue.

Boeravinone $\mathrm{A}$ forms two hydrogen bonds with docking score $-5.575 \mathrm{kCal} / \mathrm{mol}$. The hydroxyl 
group in the $9^{\text {th }}$ position of the chromeno chromon nucleus of Boeravinone A forms a hydrogen bond with $130^{\text {th }} \mathrm{GLN}$ residue (bond length-2.23410, Acceptor angle-173.152 and donor angle-164.273) Hydroxyl group in the $11^{\text {th }}$ position forms one side

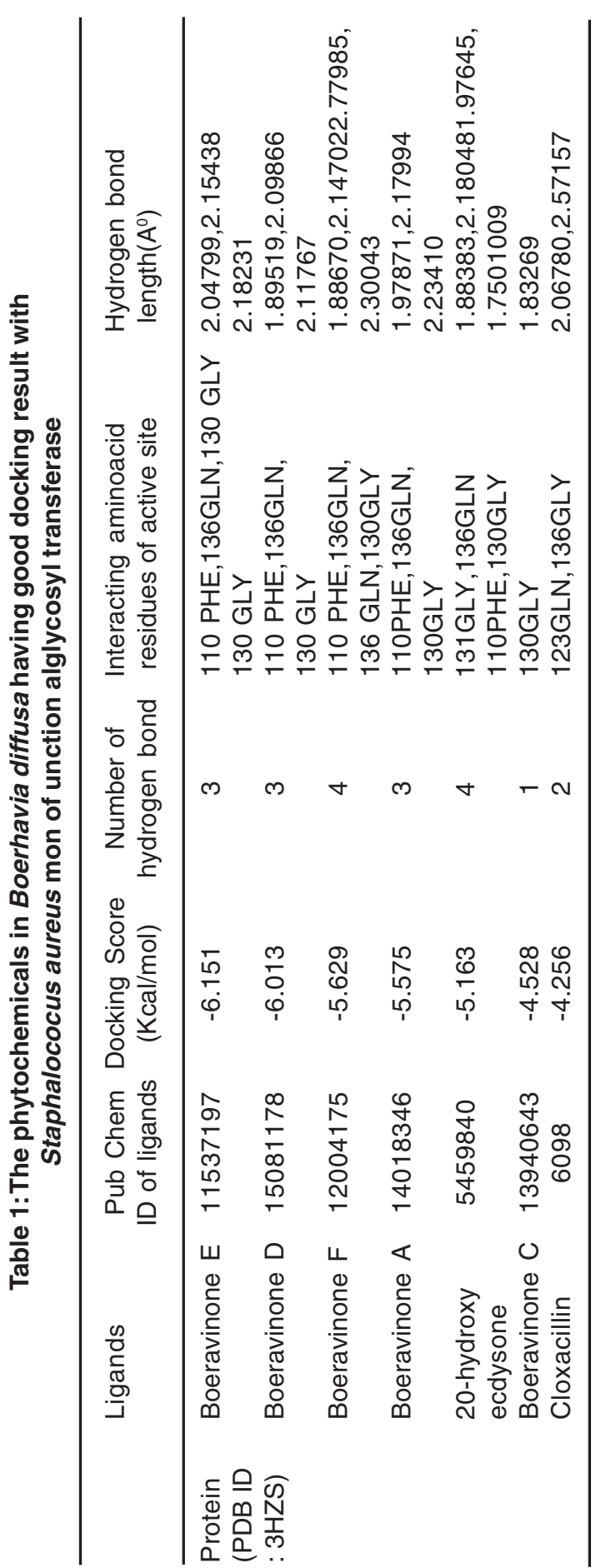

to side hydrogen bond with $110^{\text {th }} \mathrm{PHE}$. (bond length1.97871, Acceptor angle-165.258, donor angle173.134) The ketonic oxygen forms a hydrogen bond with $136^{\text {th }}$ aminoacid residue. (bondlength2.17994, Acceptor angle-158.265 and donor angle-178.365)

Boeravinone C shows a docking score $-4.528 \mathrm{kCal} / \mathrm{mol}$ and forms one hydrogen bond between the hydroxyl group and $130^{\text {th }} \mathrm{GLY}$ aminoacid residue. (bond length-1.83269, acceptor angle-165.235 and donorangle-149.245). All these components satisfied the ADME properties (Table 2).

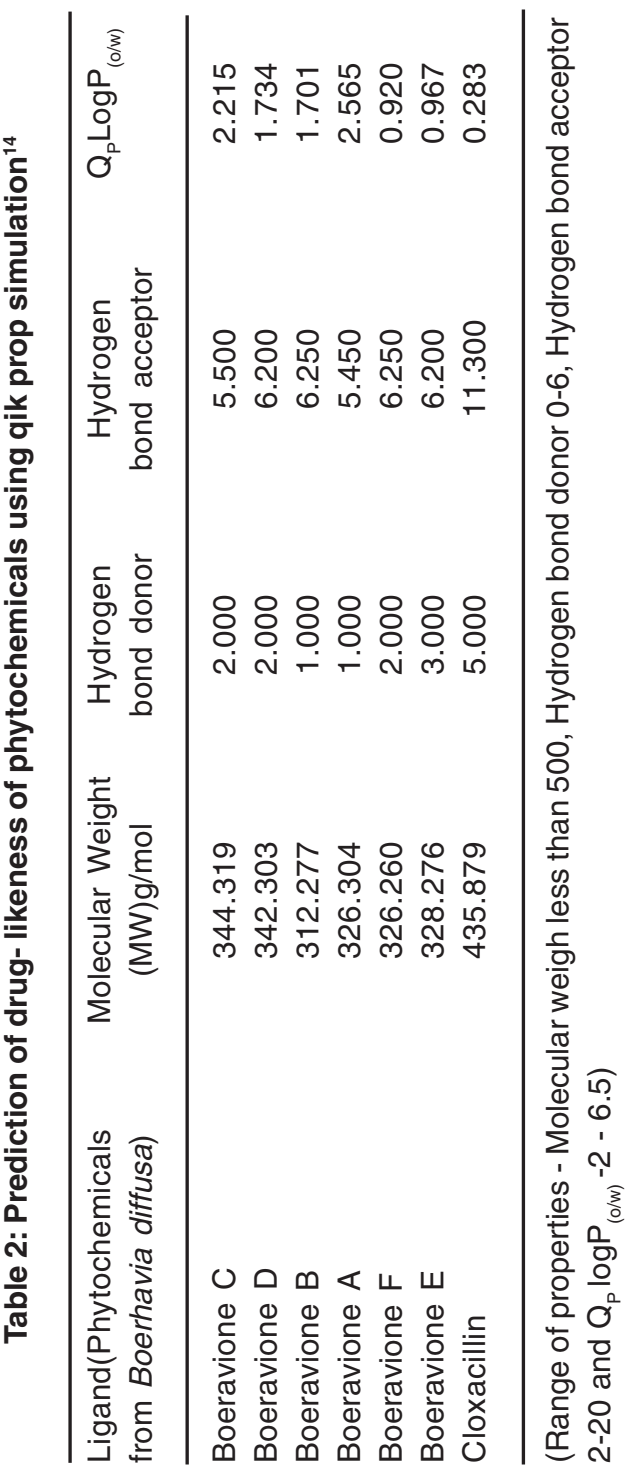


a) $3 \mathrm{HZS}$ and Boeravinone $\mathrm{E}$

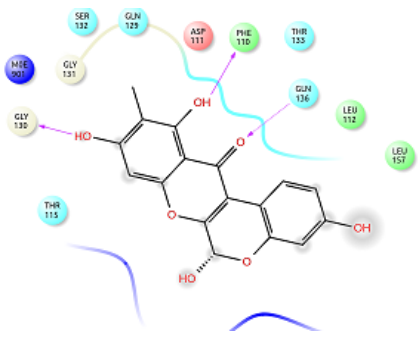

d) 3 HZS and Boeravinone A

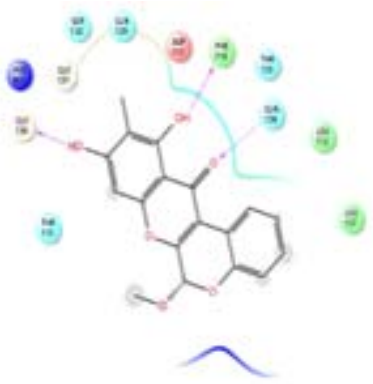

b) $3 \mathrm{HZS}$ and Boeravinone D

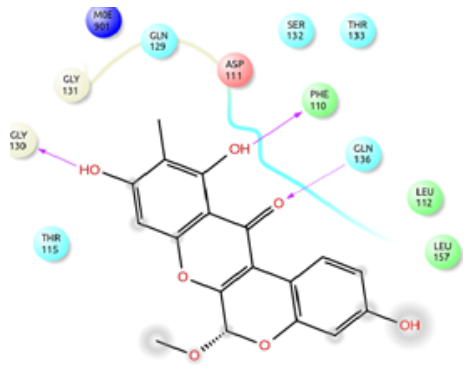

e) 3HZS and 20-hydroxyecdysone
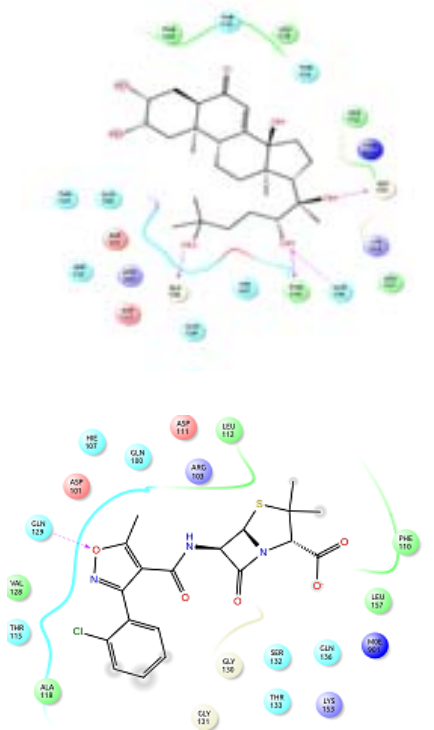

c) $3 \mathrm{HZS}$ and Boeravinone F f) 3 HZS and Boeravinone C

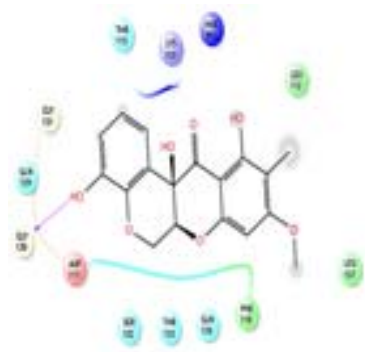

Fig. 1. 2D interaction of phytochemicals in Boerhavia diffusa satisfying ADMET properties with the target protein $3 \mathrm{HZS}$

a) $3 \mathrm{HZS}$ and Boeravinone $\mathrm{E}$

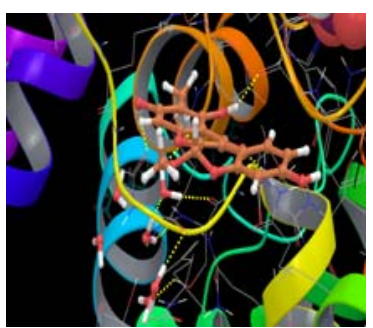

b) $3 \mathrm{HZS}$ and Boeravinone D

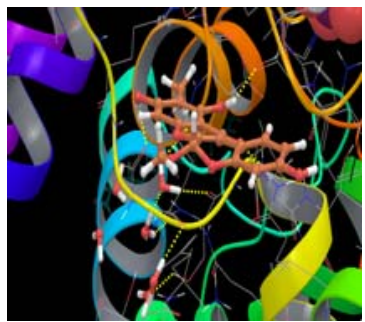

c) $3 \mathrm{HZS}$ and Boeravinone F d) 3HZS and Cloxacillin
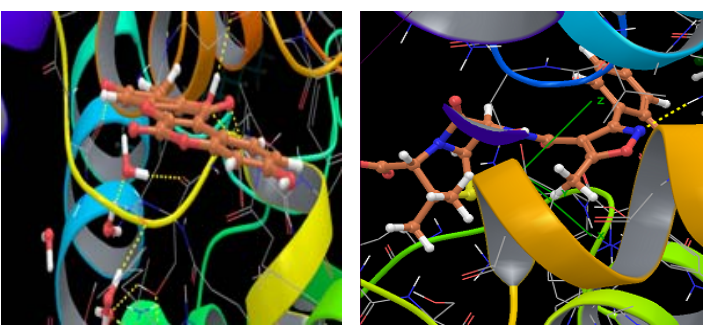

Fig. 2. 3D Molecular docking interactions of phytochemicals in Boerhavia diffusa(having most potent inhibitory activity) and Cloxacillin with Staphalococus aureus monofunctional glycosyl transferase (3HZS)

Cloxacillin shows lesser docking score $4.256 \mathrm{kCal} / \mathrm{mol}$ than the above phytochemicals with one hydrogen bond with $123^{\text {rd }}$ GLN (Bond length2.06780, Acceptor angle-165.214 and Donor angle-174.254) amino acid residue.

\section{CONCLUSION}

The phytochemicals Boeravinone $A$, Boeravinone B, Boeravinone C, Boeravinone D, Boeavinone $\mathrm{E}$ and Boeravinon $\mathrm{F}$ has potent 
inhibitory activity towards the staphalococus aureus monofunctional glycosyltransferase compared to the commercial drug Cloxacillin. These phytochemicals are responsible for the antimastitis activity of Boerhavia diffusa. This study leads to the development of novel drugs for mastitis.

\section{REFERENCES}

1. Reena Patel, J.; Prashant Kunjadia, D.; ChaitanyaJoshi, G.; Anju Kunjadia, P. International Journal of Pharmacy and Pharmaceutical sciences., 2015, 7, 496-502.

2. Wellenberg, G.J.; Van der Poel, W.H.M.; Van Orischo, J.T. Veterinary Microbiology., 2002, 88, 27- 45 .

3. Harmon, R.J. J. Dairy Sci., 1994, 77, 2103-2112.

4. Kandasamy, S.; Green, B.B.; Benjamin, A.L.; Kerr, D.E. J Dairy Sci., 2011, 12, 5963-75.

5. Department of Animal Science. "Mastitis in Dairy Cows" (PDF). MacDonald Campus of McGill University. Archived from the original (PDF) on July 8, 2003. Retrieved 4 February 2010.

6. http://en.wikipedia.org/wiki/peptidoglycan

7. Andrea FeBler;Carly Scott; Kristina Kadlec; Ralf Ehricht; Stefan Monecke; Stefan Schwarz. Journal of Antimicrobia chemotherapy., 2010, 65, 619-625.

8. Muhamed Mubarack, H.; Doss, A.; Dhanabalan, R.; Venkataswamy, R. Hygeia. J. D. Med., 2011, 3, 71-75.

9. ApurbaSarker Apu; Mahmuda Sulthana Liza; Tamaluddin, A.T.M.; Amran Howlader, M.D.; Repon Kumar Saha; Farhana Rizwan; Nishat Nasrin. Asian Pacific Journal of Tropical Biomedicine., 2012, 2, 673-678.

10. http://www.rcsb.org/pdb

11. Schrodingersuit; 2009 Protein Preparation Wizard; Epik version 2.0; Impact version 5.5. Schrodinger, LLC, New York., 2010.
12. Friesner, R.A.; Banks, J.L.; Murphy, R.B.; Halgren, T.A.; Klicic, J.J.; Maniz, D.T.; Repasky, M.P.; Knoll, E.H.; Shelley, M.; Perry, J.K.; Shaw, D.E.; Francis, P.; Shenkin, P.S. J.Med.Chem., 2004, 47, 1739-1749.

13. Babitha Agarwal; Sunanda Das; Archana Pandey. Asian journal of Applied Sciences., 2011, 3, 436-441.

14. Friesner, R.A.; Murphy, R.B.; Repasky, M.P.; Frye, L.L.; Greenwood, J.R.; Halgren, T.A.; Sanschagrin, P.C.; Maniz, D.T. J.Med.Chem., 2006, 49, 6177-6196.

15. Schrodinger suit, Protein Preparation Guide, Site Map 2.4; Glide Version 5.6,Lig Prep 2.4, Qik Propn3.3, Schrodinger, LLC, New York, NY., 2010.

16. Chude, M.A.; Orisakwe, O.E.; Afonne, O.J., Gamaniel, K.S.; Vongtau, O.H.; Obi, E. Indian Journal of Pharmacology., 2001, 33, 215-216.

17. Ujowundu, C.O.; Igwe, C.U.; Enemor, V.H.A.; Nwaogu, L.A.; Okafor, O.E. Pakisthan journal of Nutrition., 2008, 7, 90-92.

18. Meera Sumanth; Mustafa, S.S. International Journal of Pharmacology., 2007, 3, 416-420.

19. Krishnamurthy; Mayank, A.P.; Vijay Lambole. Indian Journal of experimental biology., 2010, 5, 107-110.

20. Sharma, M.; Vohra, S.; Arnason, J.T.; Hudson, J.B. Pharm Biol., 2008, 46, 11-116.

21. Akinnibosun, F.I.; Akinnibosun, H.A.; Ogedegbe, D. Science World Journal., 2009, 4, 25-28. 